\title{
Bone mineral density differences between paraplegic and quadriplegic patients: a cross-sectional study
}

\author{
S Tsuzuku*,1, Y Ikegami ${ }^{2}$ and $\mathrm{K}$ Yabe $^{2}$ \\ ${ }^{1}$ Department of Epidemiology, National Institute for Longevity Sciences, Aichi, Japan; ${ }^{2}$ Research Center of Health, \\ Physical Fitness and Sports, Nagoya University, Nagoya, Japan
}

\begin{abstract}
Study design: This cross-sectional study was conducted by comparing bone mineral density (BMD) of paraplegic and quadriplegic patients.

Objectives: The purpose of this study was to investigate the relationship between the bone mineral loss and injury level in spinal cord injury patients.

Settings: Experiments were conducted at Yoneda Hospital and Research Center of Health, Physical Fitness and Sports, Nagoya University, Nagoya, Japan.

Methods: Lumbar spine (L2-4), proximal femur (femoral neck, trochanter region and Ward's triangle) and whole body BMD were measured in ten paraplegic and ten quadriplegic patients using dual-energy X-ray absorptiometry (DXA, HITACHI BMD-1X).

Results: Significant differences were observed in the lumbar spine, trochanter region and upper extremities BMD between paraplegic and quadriplegic patients $(P<0.05, P<0.05$ and $P<0.01$, respectively), but not in the femoral neck, Ward's triangle, head, pelvis, lower extremities or whole body BMD.

Conclusion: These results suggest that the injury level influences on the lumbar spine, upper extremities and trochanter region BMD. From a biomechanical standpoint, it is possible to explain that the differences in mechanical loading exerted on bones also affected the difference of lumbar spine BMD in the two groups.
\end{abstract}

Keywords: spinal cord injury, (SCI); bone mineral density, (BMD); dual energy X-ray absorptiometry, (DXA); paraplegic patients; quadriplegic patients; mechanical loading

\section{Introduction}

Osteoporosis is a common complication of spinal cord injury (SCI). ${ }^{1-4}$ As fractures derived from osteoporosis frequently occur in SCI patients during daily life and rehabilitation programs ${ }^{5}$ prevention of bone mineral loss afer SCI is important. Biering-Sorensen et $a l^{1}$ observed bone mineral loss in the paralysed limbs of SCI patients in a 3-year prospective study, mainly in the femoral neck and the proximal tibia. Garland et $a l^{4}$ also reported that most of the bone loss occurred rapidly during the first 4 months and stabilized after $12-16$ months to two thirds of the original bone mass. These results suggest that demineralization in SCI patients is caused by immobilization and disuse due to paralysis. Although these studies demonstrated that the demineralization occurred rapidly in the paralyzed area after injury, the mechanism of bone mineral loss was not clearly described.

Our previous study showed that high intensity compressive stress effectively increased the lumbar

*Correspondence: S Tsuzuku, 36-3 Gengo, Morioka-cho, Obu-city, Aichi pref, 474-8522 Japan spine and whole body bone mineral density (BMD) in healthy male powerlifters. ${ }^{6}$ Granhed et $a l^{7}$ also found that bone mineral content (BMC) of the lumbar vertebra in powerlifters was significantly higher than in controls and also biomechanically estimated that the load on the third lumbar vertebra during a deadlift exercise was $18.8-36.4$ kilo newton $(\mathrm{kN})$. These results suggest that the strain magnitude, site specificity, and distribution of strain throughout the bone structure are important factors in the adaptive response of bone. ${ }^{8}$

Our SCI patients were divided into two groups (paraplegia and quadriplegia) according to their injury level. Paraplegia is paralysis of the lower part of the body, including both legs. In contrast, quadriplegia refers to paralysis of the upper and lower body, including both arms and legs. The paralyzed area may markedly influence the BMD, but few studies have focused on the relationship between the injury level and bone mineral loss.

In this study, the BMD of SCI patients was measured and the possible mechanisms which affect the difference in bone mineral loss were discussed by comparing the BMD of paraplegic and quadriplegic patients. 


\section{Methods}

\section{Subjects}

Twenty men with SCI participated in this study, ten with quadriplegia and ten with paraplegia. All subjects were wheelchair dependent, had no history of metabolic bone disease and were not taking medication known to affect mineral metabolism as assessed by self-reports. All subjects signed an informed consent statement. Table 1 shows the characteristics of the subjects. There were significant differences in both age and the post traumatic period between the two groups. Circumferences of the neck, chest, upper arm and forearm were measured using standard anthropometric measurement methods. Details of the subjects are summarized in Table 2 .

\section{BMD measurement}

The BMD of the lumbar spine (L2-4), proximal femur (femoral neck, trochanter region and Ward's triangle) and the whole body were measured by dual-energy Xray absorptiometry (DXA, HITACHI BMD-1X). The BMD of the head, upper extremities, trunk, ribs, pelvis, spine and lower extremities were also measured by a whole body scan. All scanning and analyses were performed by the same operator to assure consistency. The day-to-day precision (coefficient of variation; $\mathrm{CV}$ ) of the BMD measurement was $0.7 \%$.

\section{Statistical methods}

All statistical analyses were conducted with a Statview 4.5 (Abacus Concepts, Inc., Berkeley, CA, USA) on a Macintosh computer. Statistical significance of differ- ences between the two groups was determined using the Student's $t$-test. All comparisons were considered statistically significant if $P<0.05$.

\section{Results}

As analyzed by the Student's $t$-test (Figure 1), the BMD of the lumbar spine, upper extremities and trochanter region was significantly higher in the paraplegic group (PG) than in the quadriplegic group (QG). However, no significant differences were found in the femoral neck, Ward's triangle, head, pelvis, lower extremities or whole body BMD. PG showed significantly larger circumferences in the upper body than the QG, but no significant differences were observed in the neck (Table 1).

\section{Discussion}

In this study, significant differences were found in the lumbar spine, trochanter region and upper extremities BMD between paraplegic and quadriplegic patients, but not in the femoral neck, Ward's triangle, head, pelvis, lower extremities or whole body. BieringSorensen et $a l^{1}$ found normal BMD in the lumbar spine and demineralization in the femoral neck, shaft and the proximal tibia in paralyzed SCI patients. The maintenance of normal lumbar spine BMD after SCI may be due to continued weight bearing during wheelchair use. Although our results of lumbar spine BMD in the QG contradict previously published data, $, 1,2,9,10$ most research focused on BMD in paraplegic patients. Moreover, Lanyon et al ${ }^{11}$ showed the importance of mechanical loading and site specificity for maintaining or increasing BMD. These

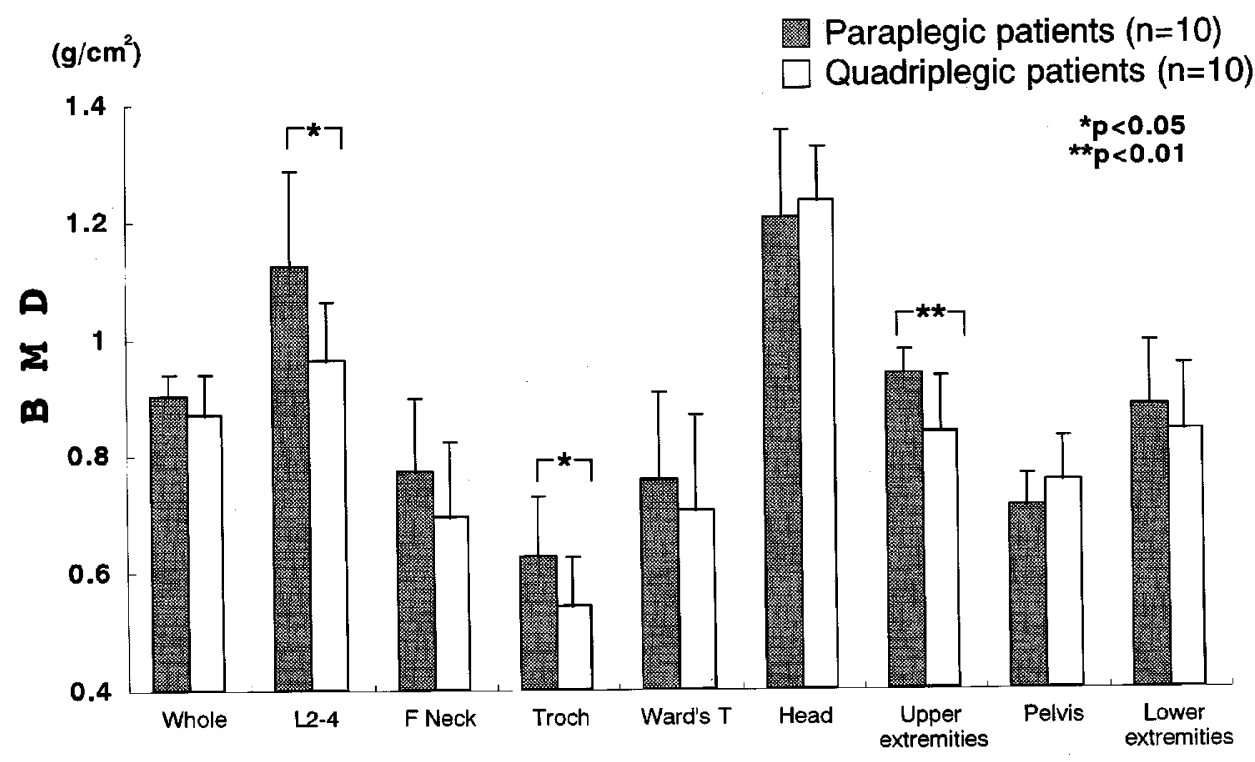

Figure 1 Bone mineral density in paraplegic $(n=10)$ and quadriplegic patients $(n=10)$. Whole: whole body, L2-4: lumbar spine 2-4, F Neck: femoral neck, Troch: trochanter region, Ward's T: Ward's triangle. Values are expressed as mean \pm SD 
results suggest that the injury level influences the BMD and the magnitude of the mechanical loading on the lumbar spine. The mechanical model shown in Figure 2 (left) was considered to analyze the force on the lumbar spine while sitting in a wheelchair with slightly forward inclination which is the most common posture during desk work. To maintain constant inclination of the trunk segment, a force necessitating backward rotation of the trunk segment should be applied. This force must be supplied by muscle contraction of the erector spinal muscle group and multifidus and is partly observed in paraplegic patients. Thus the forces hold this posture in balance. To consider this balance simply, we can view the spinal column as a rigid body and the force as the weight of the upper body and

Table 1 Characteristics and anthropometric data of paraplegic and quadriplegic patients (mean $\pm \mathrm{SD}$ )

\begin{tabular}{lccc}
\hline & $\begin{array}{c}\text { Paraplegic } \\
(\mathrm{n}=10)\end{array}$ & $\begin{array}{c}\text { Quadriplegic } \\
(\mathrm{n}=10)\end{array}$ & \\
\hline Age (years) & $44.1 \pm 14.3$ & $30.2 \pm 9.0$ & $*$ \\
Body weight (kg) & $57.4 \pm 4.1$ & $55.5 \pm 6.5$ & - \\
Post traumatic & $16.1 \pm 10.1$ & $7.9 \pm 3.5$ & $* *$ \\
$\quad$ period (years) & & & \\
Chest (cm) & $90.2 \pm 5.1$ & $84.7 \pm 4.5$ & $* *$ \\
Right upper & $29.4 \pm 2.2$ & $25.1 \pm 2.8$ & $*$ \\
$\quad$ arm (cm) & & & $* *$ \\
Right forearm (cm) & $26.0 \pm 1.0$ & $23.0 \pm 1.5$ & $* *$ \\
Neck (cm) & $37.5 \pm 2.0$ & $36.2 \pm 1.8$ & - \\
\hline
\end{tabular}

muscle contraction applied at the top of the spinal column. The resultant force is the vector sum of the force from the weight of the upper body $(\mathrm{FW})$ and the force generated by the erector spinal muscle group and the multifidus muscle (FE). Its application line is along the long axis of the spinal column. This resultant force

Table 2 Details of the 20 subjects

\begin{tabular}{lcccc}
\hline $\begin{array}{l}\text { Participant } \\
\text { no. }\end{array}$ & $\begin{array}{c}\text { Age } \\
\text { years) }\end{array}$ & $\begin{array}{c}\text { Post traumatic } \\
\text { period }\end{array}$ (years) & $\begin{array}{c}\text { Level of injury } \\
\text { Incomplete }\end{array}$ & \begin{tabular}{c} 
Complete \\
\hline 1
\end{tabular} \\
34 & 6.5 & C4 & C5 \\
2 & 23 & 7 & & C5 \\
3 & 17 & 3 & & C5 \\
4 & 28 & 6.5 & C6 & C8 \\
5 & 39 & 11.5 & & C6 \\
6 & 23 & 3 & & C6 \\
7 & 35 & 11 & C6 & C8 \\
8 & 48 & 13 & & C7 \\
9 & 27 & 7 & & C7 \\
10 & 28 & 10.5 & & C8 \\
11 & 40 & 21 & & Th11 \\
12 & 59 & 23 & & Th11 \\
13 & 60 & 23 & & Th11 \\
14 & 31 & 3.5 & & Th11 \\
15 & 36 & 4.5 & & Th12 \\
16 & 54 & 24.5 & & Th12 \\
17 & 31 & 5.5 & & L1 \\
18 & 20 & 4.5 & & L1 \\
19 & 52 & 22.5 & & L1 \\
20 & 58 & 28.5 & & L2 \\
\hline
\end{tabular}
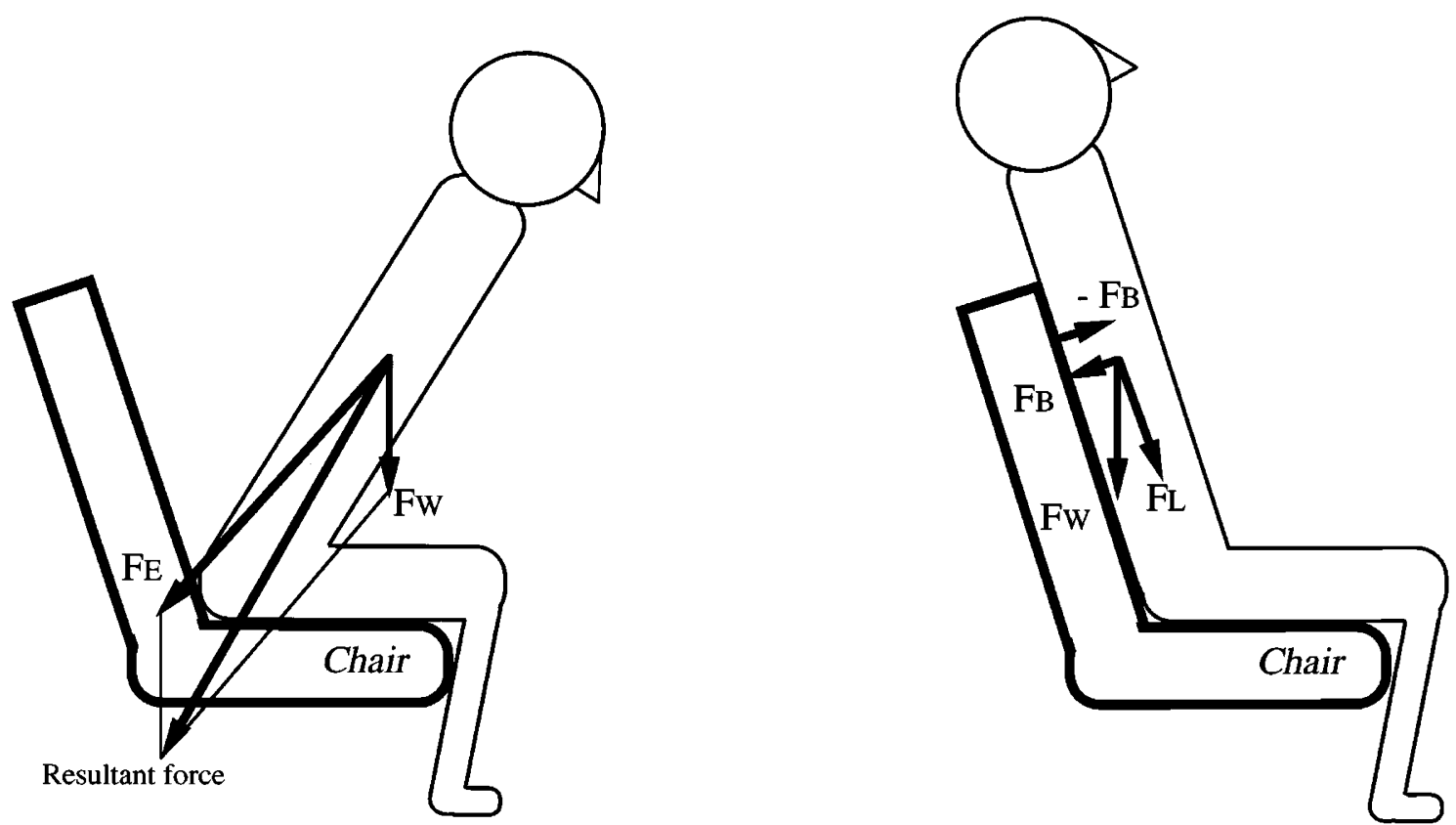

Figure 2 Schematic view of sitting with slightly forward inclination (left) and leaning back in the chair (right). Fw: force from weight of the upper body, FE: force generated by the erector spinal muscle group and multifidus muscle, FL: force on the lumbar spine, FB: force toward the back 
is applied as compressive stress and its magnitude is much larger than the $\mathrm{FW}$. This compressive stress may affect the lumbar spine BMD (Figure 2 left). Quadriplegic patients cannot use the erector spinal muscle group or multifidus muscle due to their injury level, so they tend to lean back in their wheelchair. The right side of Figure 2 shows that the force from the weight of the upper body is divided into two forces, the force on the lumbar spine (FL) and the force toward the back $(\mathrm{FB})$ while leaning back in the wheelchair. FL is smaller than Fw. Hence, the great compressive stress seen in the PG was not present in the QG. These findings suggest that mechanical loading on the lumbar vertebra affects the lumbar spine BMD. Our previous study demonstrated a significant relationship between the BMD and the magnitude of mechanical loading in the lumbar spine and supports our present hypothesis.

Kerr et $a l^{12}$ examined the effect of exercise on bone mass in postmenopausal women and observed significant increase in the BMD of the trochanter region where various muscle groups were attached but not in the BMD of the femoral neck where no muscles were attached. They speculated that these results were due to muscle pull which is mediated through the force of muscle contraction at the site of attachment of tendon to bone. Thus, the bone may respond locally to reallocate the forces generated from the muscle at the loading site.

In the PG, which maintains some erector spinal muscle group and/or multifidus muscle function, muscle pull occurred in the lumbar region. In contrast, in the QG, which cannot activate the erector spinal muscle group of multifidus muscle function due to the injury level, no muscle pull occurred in the lumbar region. The lumbar spine BMD differences between the PG and the QG in this study may be derived from not only the compressive stress on the lumbar spine, but also the muscle pull.

Furthermore, previous studies have reported that bone mineral loss was clearly observed in older patients and depended on the post traumatic period. ${ }^{3,4,13,14}$ In this study, although mean age of the PG was higher and the mean post injury period was longer than the QG, BMD of the PG was significantly higher in the lumbar spine, upper extremities and trochanter region than in the QG.

Significant differences were detected in circumferences between the PG and the QG. Paraplegic patients can use their arms and therefore maintain the girth of the upper arm and forearm. Furthermore, lifetime tennis players showed greater BMD and circumferences in the dominant forearm compared with the nondominant forearm. ${ }^{15,16}$ These studies suggest that immobilization and disuse are associated with decreases in BMD and muscle size. Therefore, the differences in circumferences between the two groups may have been caused by differences in the injury level and immobilization.

In this study, the trochanter region's BMD of the PG was also significantly higher than the QG.
Although lateral vastus, piriform, gluteus medius and gluteus minimum muscles attach to the trochanter region, both the $\mathrm{PG}$ and the $\mathrm{QG}$ have no muscle functions due to the injury level. Furthermore, as both groups are wheelchair dependent, there is no significant difference concerning the mechanical loading due to their body weight. This result could be explained by neither immobilization, mechanical loading nor muscle pull.

In conclusion, the BMD of SCI patients varied with the injury level. Mechanical loading and muscle pull, which depend on their injury level, may affect the variation of bone mineral loss in SCI patients.

\section{Acknowledgements}

A part of this study was financially supported by the Ministry of Education, Science, Sports and Culture, Grant No. 07458016. We are grateful to Dr Minoru Yoneda for his support.

\section{References}

1 Biering-Sorensen F, Bohr HH, Schaadt OP. Longitudinal study of bone mineral content in the lumbar spine, the forearm and the lower extremities after spinal cord injury. Europ J Clin Invest 1991; 20: $330-335$.

2 Biering-Sorensen F, Bohr HH, Schaadt OP. Bone mineral content of the lumbar spine and lower extremities years after spinal cord lesions. Paraplegia 1988; 26: $293-301$.

3 Wilmet E et al. Longitudinal study of the bone mineral content and of soft tissue composition after spinal cord section. Paraplegia 1995; 33: 674-677.

4 Garland DE et al. Osteoporosis after spinal cord injury. J Orthop Res 1992; 10: $371-378$.

5 Comarr AE, Hutchinson RH, Bors E. Extremity fractures of patients with spinal cord injuries. Am J Surg 1962; 103: $732-739$.

6 Tsuzuku S, Ikegami Y, Yabe K. Effects of high-intensity resistance training on bone mineral density in young male powerlifters. Calcif Tissue Int 1998; 63: 283-286.

7 Granhed H, Jonson R, Hansson T. The loads on the lumbar spine during extreme weight lifting. Spine 1987; 12: 146-149.

8 Lanyon LE, Rubin C, Baust G. Modulation of bone loss during calcium insufficiency by controlled dynamic loading. Calcif Tissue Int 1986; 38: 209-216.

9 Uebelhart D et al. Early modifications of biochemical markers of bone metabolism in spinal cord injury patients. A preliminary study. Scandinavian Journal of Rehabilitation Medicine 1994; 26: $197-202$.

10 Leslie WD, Nance PW. Dissociated hip and spine demineralization: a specific finding in spinal cord injury. Archives of Physical Medicine \& Rehabilitation 1993; 74: $960-964$

11 Lanyon LE, Rubin CT, Baust G. Modulation of bone loss during calcium insufficiency by controlled dynamic loading. Calcif Tissue Int 1986; 38: 209-216.

12 Kerr D, Morton A, Dick I, Prince R. Exercise effects on bone mass is postmenopausal women are site-specific and loaddependent. J Bone Miner Res 1996; 11: 218 - 225.

13 Changlai SP, Kao CH. Bone mineral density in patients with spinal cord injuries. Nucl Med Commun 1996; 17: 385-388.

14 Davis JW et al. Comparison of cross-sectional and longitudinal measurements of age-related changes in bone mineral content. $J$ Bone Miner Res 1989; 4: 351 - 357.

15 Huddleston AL, Rockwell D, Kulund DN, Harrison RB. Bone mass in lifetime tennis athletes. JAMA 1980; 244: $1107-1109$.

16 Jones HH et al. Humeral hypertrophy in response to exercise. $J$ Bone Joint Surg 1977; 59A: 204-208. 